\title{
POWER REQUIREMENTS AND MECHANICAL EFFICIENCY OF TREADMILL WALKING*†
}

\author{
M. Y. ZARRUGH + \\ Department of Mechanical Engineering and Applied Mechanics, \\ University of Michigan, Ann Arbor, MI 48109, U.S.A.
}

\begin{abstract}
The instantaneous energy levels of body segments are computed from kinematic measurements on a level treadmill at various speeds with freely chosen step rates and a constant speed with different imposed step rates. The changes in the energy levels of segments are combined to compute the average work rate required to accelerate the total body (positive internal work). This work is compared to total metabolic power consumption to obtain a minimal estimate of mechanical efficiency. The efficiency increases rapidly from $9 \%$ to $0.84 \mathrm{~m} / \mathrm{sec}$ to a maximum of $23 \%$ at $1.70 \mathrm{~m} / \mathrm{sec}$. Thereafter, the efficiency slowly decreases with speed to $18 \%$ at $2.35 \mathrm{~m} / \mathrm{sec}$. When different step rates are imposed at one constant speed, the average positive work rate remains constant. This work rate level is identical to that required for walking at the same speed with self-determined (free) step rate. Thus, maximum gross efficiency results at the free step rate.
\end{abstract}

\section{INTRODUCTION}

Assessment of energy requirements of various body segments provides a basis for evaluating energy needs of prostheses and yields valuable understanding of how muscles perform mechanical work in locomotion. A complete energy analysis must include not only the work done to change the energy levels of segments but also the work done at the joints. The first type of work is determined from changes in the energy levels that can be computed from purely kinematic measurements such as those reported in Zarrugh and Radcliffe (1979). The work done at the joints is computed from loads and relative motions at the joints.

In walking, the work done is derived from internal sources and does not involve the exchange of mechanical energy between the body and the environment, if air resistance and friction losses at the feet are neglected. Thus, it is confusing to refer to the work done in locomotion as external work (Fenn, 1930a; Cavagna et al., 1963). Winter (1979) has provided a rigorous and rational definition of work performed during locomotion. He proposed dividing this work into internal work required to accelerate body segments and external work done against an external force as in lifting. pushing or pulling a load.

Determination of the energy level of body segments requires the complete specification of the motions of the center of mass of every segment. Fenn (1930a, b)

\footnotetext{
* Received 28 August 1979; in revised form 2 September 1980

+ Major conclusions regarding efficiency were presented in an abbreviated oral form at the Second Annual Meeting of the American Society of Biomechanics, October 1978, Ann Arbor, MI. See Zarrugh (1978) reference.

† Assistant Professor of Mechanical Engineering and Applied Mechanics.
}

determined the work done by the body in running through motion-picture recording of body displacements and measurements of foot pressure. Using a similar technique, Elftman $(1939,1940)$ studied energy changes and work done in the body during walking and running. While Fenn and Elftman based their energy computations on kinematic data from a single walking cycle for a single individual, Bresler and Berry (1951) determined the average energy requirements of a group of four normal subjects on the basis of displacements recorded with motion-picture cameras. Later Bresler et al. (1957) considered the energy and power requirements of the legs of four above-knee amputees wearing various prostheses. They concluded that functional improvements in the prosthetic leg, which reduce the irregularity of the trunk motion, might result in substantial reduction in the total energy needs.

Cavagna and co-workers $(1963,1966,1975)$ computed the so-called "external" work required to increase the translational mechanical energy of the body's center of mass. This "external" work was determined from accelerometer records of the trunk and motion picture records of the limbs (Cavagna $e t$ al., 1963) or by direct integration of force plate records which eliminated the need for measuring segment motions (Cavagna and Margaria, 1966; Cavagna, 1975). Gersten et al. (1969) computed "external" work in a manner similar to Cavagna et al. (1963) except that no correction was made for the difference between accelerations of the trunk and the body. The above approach underestimates the energy requirements of the body because the energy associated with opposing motions of the two lower limbs is not reflected in the energy requirements computed directly from the motion of the common center of mass.

A direct method of measuring energy levels of body segments was described by Lukin et al. (1967) and 
Raiston and Lukin (1969). The method involves computing energy from motions measured by strings attached to the approximate location of the center of mass of principal body segments.

Winter et al. (1976) provided a detailed analysis of instantaneous energy requirements of a normal subject during walking at one speed. Their segment-bysegment analysis which was based on displacement data recorded by a television-computer system considered all energy components and exchanges between segments. Some major energy parameters were also reported for four other normal subjects.

If the rate of total (internal plus external) work done to accelerate the body is compared to gross metabolic power expenditure, a gross mechanical efficiency results (Zarrugh, 1976, 1978; Winter, 1979). Although this efficiency reflects only positive work which requires much greater metabolic power than negative work (Abbott et al., 1952), negative work can also be included, if the relative metabolic cost of positive and negative work are known or assumed. Thus, the efficiency computed by considering only positive work represents a low estimate of the true value. Ralston and Lukin (1969) reported a gross efficiency of $21-24 \%$ during treadmill level walking at "comfortable" speeds. When both external and internal work [using the definition of Winter (1979)] are performed as in grade walking and bicycling and cranking against an external load, the gross efficiency increases from 11 to $20 \%$ with increased load (Bobbert, 1960). This variation with work load is expected since a larger and larger fraction of the metabolic energy is absorbed by external work.

\section{METHOD AND FORMULATION}

In this paper, the kinematic data reported in Zarrugh and Radcliffe (1979) are used to compute the energy levels of major body segments for several walking speeds ranging from 0.9 to $2.2 \mathrm{~m} / \mathrm{sec}$ on a level treadmill at step rates chosen by the subject (free walking). Energy-work exchanges are also considered under the condition of forcing of different step rates (90-140 steps/min) at a constant speed (forced walking). The results of one typical subject who is well accustomed to treadmill walking are reported.

For the purpose of the analysis, the body is divided into seven rigid components: HAT (Head-ArmsTrunk) and three segments, thigh, shank and foot, for each of the lower limbs. The absolute motion of each of these divisions was computed from displacements measured using string transducers and self-aligning electro-goniometers. Three pairs of strings were used to observe the absolute position of the HAT in space. Relative rotations at the joints of the right limb were measured using three sets of three-dimensional goniometers at the hip, knee and ankle. The kinematic data, as well as the displacement of the treadmill, were gathered at a rate of 200 samples/sec for 32 steps at each step rate used. Data was averaged to obtain mean values of all measurements. Prior to any further reduction, data were filtered by harmonic truncation at the twelfth harmonic. The error associated with reduced measured data was not expected to exceed $6 \%$.

The mixed set of absolute and relative measurements were converted into absolute three-dimensional motion data of the mass centers as detailed in Zarrugh and Radcliffe (1979). In order to compute kinetic energy, the speed of the treadmill was added to the speed of each segment. The treadmill was equipped with heavy flywheels to insure minimum belt speed variation. This variation was documented by $\mathrm{La}$ moreux (1971) to be within $2 \%$. An error of about $8 \%$ is associated with computed segment velocities and $16 \%$ with computed energy values.

The mechanical energy level, ME, of a segment is the sum of its kinetic energy, KE, and its gravitational potential energy, PE. The KE term includes both translational and rotational components. The energy formulation adopted in this paper is essentially identical to that used by Bresler and Berry (1951) and Winter et al. (1976), except that matrix methods were used to transform the principal moments of inertia, referred to the axes of symmetry of the segment, to those referred to the fixed reference axes.

The average positive mechanical power required to accelerate each body segment is computed by differentiation of energy levels. The differentiation scheme was based on the use of 12 harmonic coefficients. During both free and forced walking, the average positive power requirements of the body were computed as the sum of those of the components. These were then divided by the metabolic power expended, to find the mechanical efficiency at each speed or step rate. The metabolic energy expenditure rates were measured simultaneously with motion data. Details of the oxygen consumption-based procedure appeared in Zarrugh and Radcliffe (1978). The subject used in this study is subject $A$ in the 1978 study. The efficiency values reported here should be considered as lower bounds since negative work was not taken into account.

\section{RESULTS AND DISCUSSION}

\section{Energy level of "free" gait}

The total mechanical energy levels of the body and its various divisions are shown in Figs. 1(a)-(f) for a typical subject [see Zarrugh and Radcliffe (1979) for anthropometric data] walking at several speeds with freely selected step rates of $90-130$ steps/min. The energy level of the body as a whole, as well as its power requirements, were also computed using the assumption of symmetry between the two lower limbs. Symmetry means that except for the phase difference, the two limbs have identical motions. Generally, the results are similar to those reported by Bressler and Berry (1951), Ralston and Lukin (1969) and Winter et 

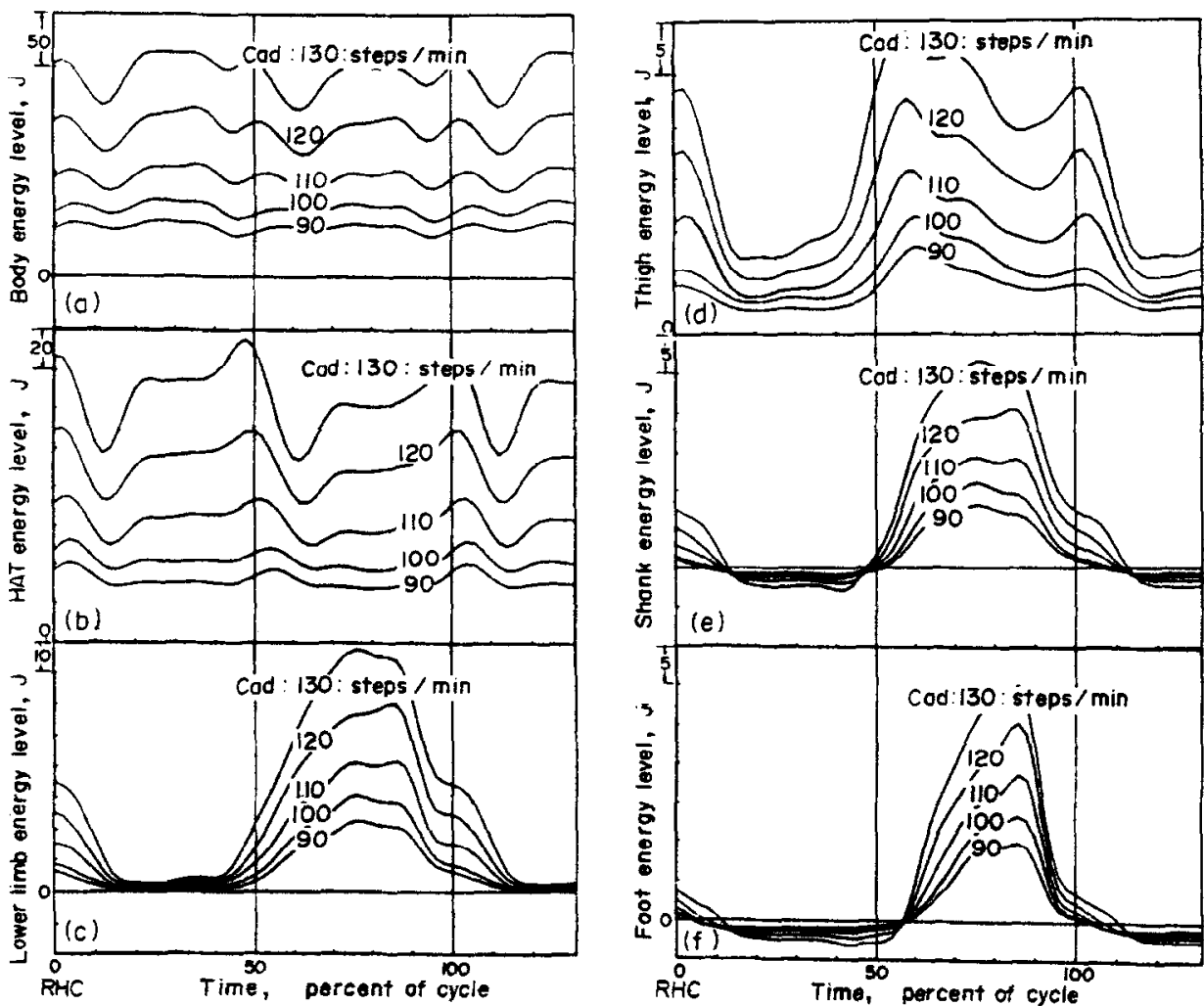

Fig. 1. Instantaneous energy levels of the body and its divisions in free walking. For this subject, the walking speed in $\mathrm{m} / \mathrm{sec}$ can be obtained by dividing the cadence squared in (steps/min) ${ }^{2}$ by 8000 , e.g. (110 steps $/ \mathrm{min})^{2} / 8000=1.51 \mathrm{~m} / \mathrm{sec}$.

al. (1976). However, the results in this report present a clearer demonstration of the effects of speed on energy and power levels. Figure 1(a) shows that the energy level of the body is fairly constant at low step rates. As cadence increases, the body undergoes increased energy level fluctuations. The largest changes occur during double-support phase, when support is transferred to one leg while the other leg prepares for pushoff. The relative contribution of major body divisions to the total energy level of the body is more clearly shown in Fig. 2. Major energy changes in the segments of the lower limb occur predominantly during swing phase as depicted in Figs. 1(c)-(f) for the right side. During single-support phase, the right limb maintains a low energy level until late right stance phase when the level increases rapidly in preparation for swing (Fig. 1c). This period, named the "tranquility" period by Elftman (1955), is present in all lower limb segments (Figs. 1d-f). These figures also indicate that peak muscular activity that accelerates the segments occurs first at the hip followed by the knee and the ankle.

Mechanical energy components of the body and its divisions are displaye, in Figs. 3(a)-(f) at an intermediate cadence of $110 \mathrm{steps} / \mathrm{min}$. The kinetic energy is the largest contributor to energy of the body and HAT (Figs. $3 a$ and $b$ ). The same figures show that the rotatory kinetic energy, $\mathrm{KE}$, is never larger than $1 \%$ of the total ME of the body and HAT. The RE term is largest for the thigh and the shank (Figs. 3d and 3e), peaking at $6 \%$ of the maximum ME. Similar observations were reported in other studies, such as Winter et al. (1976) and Bresler and Berry (1951). Thus, the RE terms and their derivatives are considered to be of minor importance and can be ignored, if desired, without introducing a large error.

Since the energy changes in the leg are almost entirely kinetic in nature, the body's KE changes

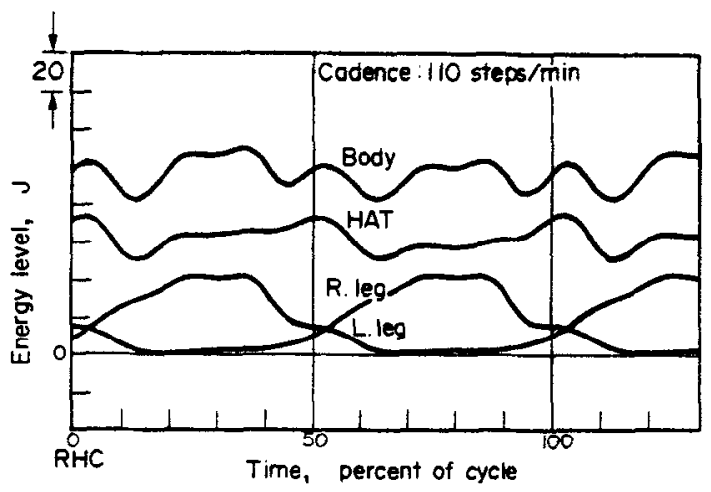

Fig. 2. Contribution of major body segments to the total energy level. 

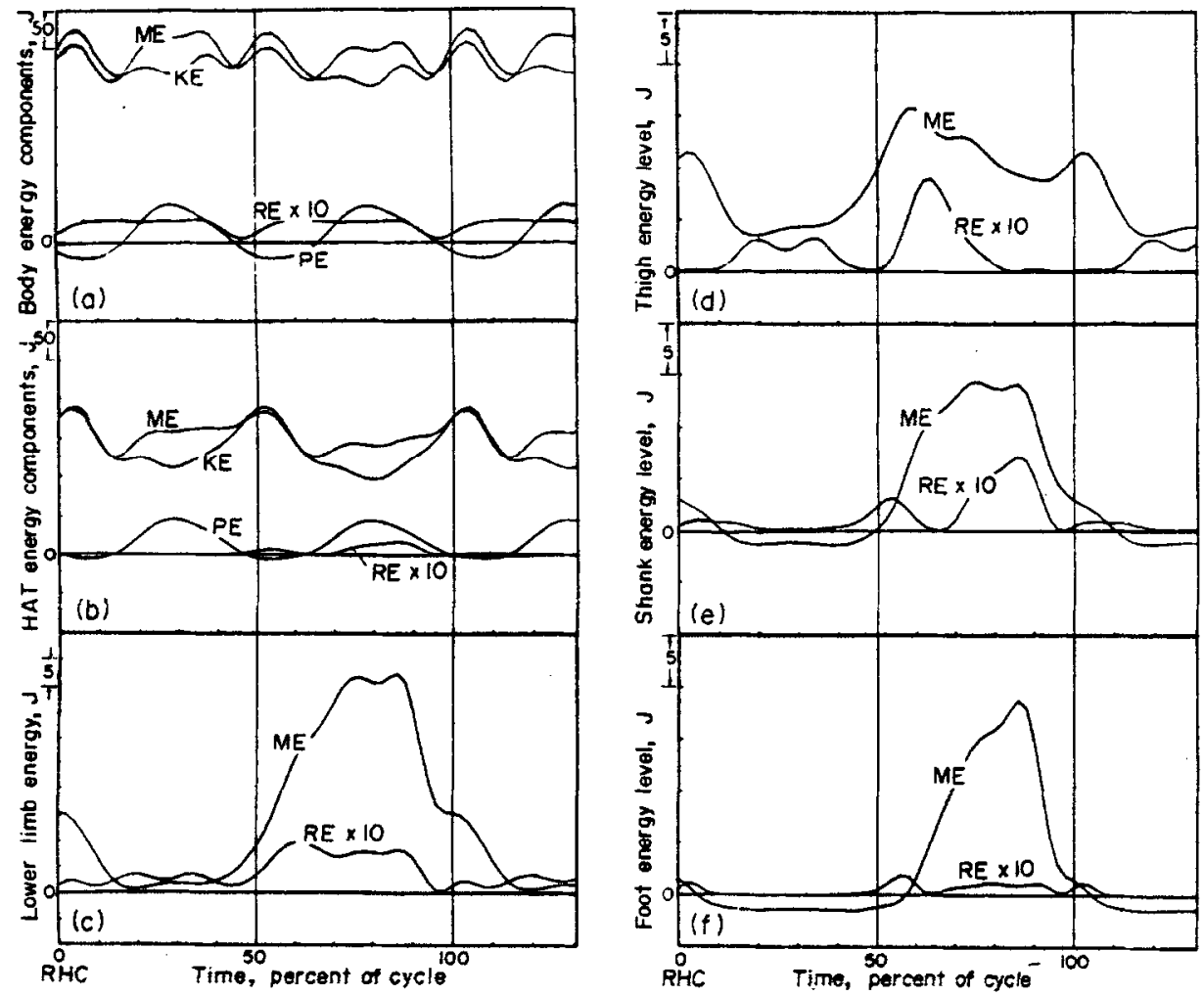

Fig. 3. Mechanical energy components of the body and its segments at an intermediate speed of $1.5 \mathrm{~m} / \mathrm{sec}$ corresponding to a cadence of 110 steps/min. ME-total mechanical energy. KE-translational kinetic energy. RE-rotatory kinetic energy. PE-potential energy.

generally exceed its PE changes resulting in a relatively unsteady energy level. In contrast, the HAT's KE and PE changes tend to cancel each other giving it a more steady energy level than the body. Winter (1979) points out that this is the main reason for the discrepancy in energy changes predicted on the basis of the motion of the body's common center of mass as opposed to those based on the sum of the energy of segments; the mass center has a total energy schedule similar to that of the HAT which does not reflect the contribution of the lower limbs.

\section{Power requirements of segments in free walking}

The instantaneous rate of change of the mechanical energy level of any segment indicates the power required to accelerate or decelerate the segment at that instant of time. Positive power accelerates the segment, while negative power decelerates it. Figures $4(a)-(f)$ display the power requirements of the body and its various divisions. In computing the power requirements of the body as a whole, perfect exchange of energy is assumed to take place between various body segments. This means that the total energy of the body is equal to the unweighted sum of the mechanical energy of the parts.

The peak power requirements of the body occur during double-support phase (Fig. 4a). These peaks correspond to similar ones in the HAT (Fig. 4b) and the lower limbs (Fig. 4c). The power curves of the body and the HAT are very similar in shape except in late swing when the body experiences an opposite power flow to that of the HAT due to major decelerations of the lower limbs in preparation for heel contact.

The analysis of power requirements of the body and its major divisions is shown in Fig. 5. The power flows in the legs are in opposite directions when one limb accelerates in preparation for swing and the other decelerates in preparation for heel contact. When combined, these flows cancel each other and reduce the power requirements of the body (Fig. 5).

\section{Energy levels in forced walking}

It was shown that when people are allowed to choose a step rate to walk at a prescribed speed, they choose one which results in a minimum average metabolic power consumption (Zarrugh and Radcliffe, 1978). If they are forced to walk with any other step rate, at the same prescribed speed, an increased metabolic cost results. Identification of this optimization process is possible only through the consideration of the forced walking pattern. Energy and power are considered in forced walking to determine whether or not mechanical power is also minimized.

The energy levels of the body and its various components are shown in Figs. 6(a)-(f). These levels were computed from kinematic data of the forced 

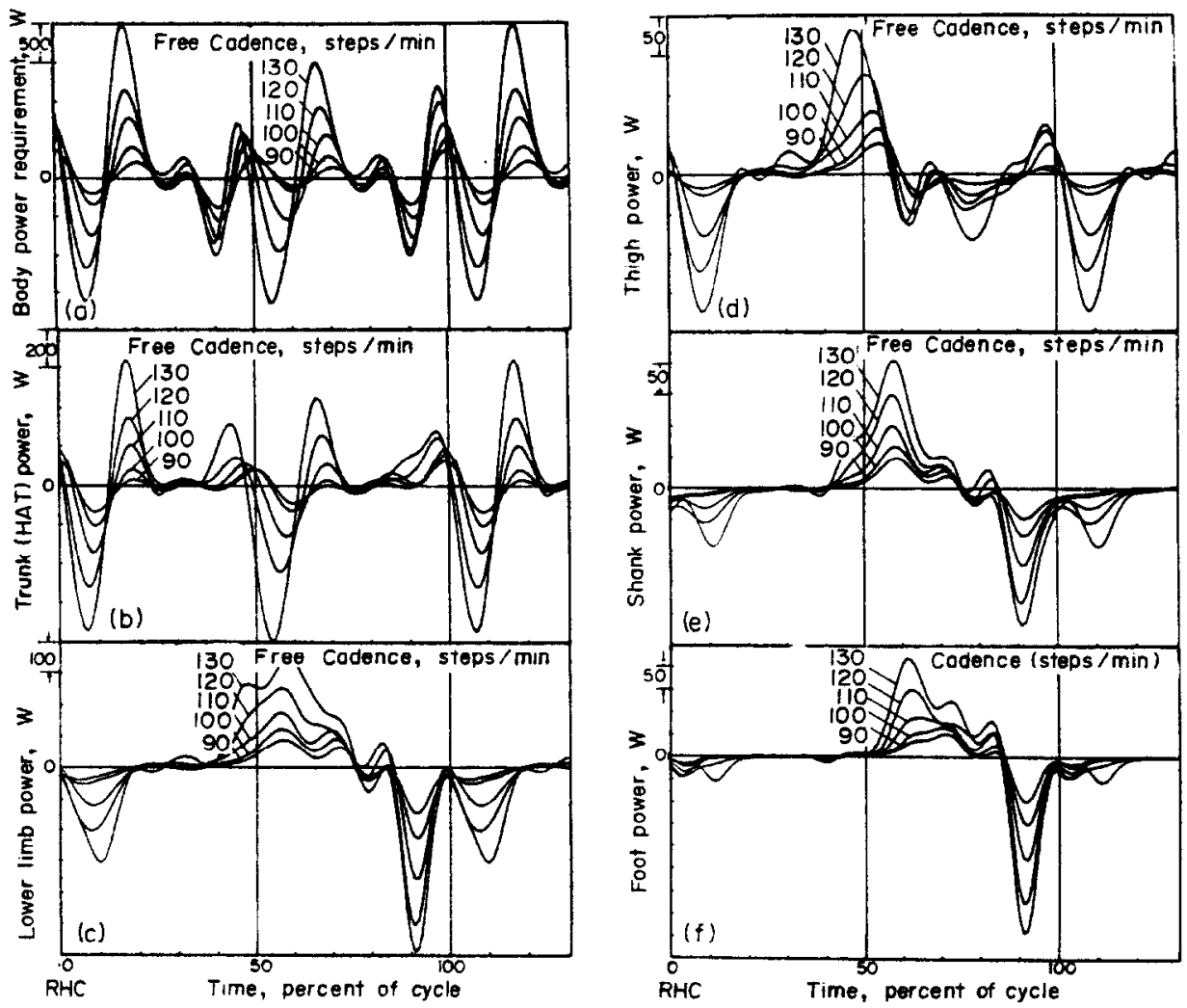

Fig. 4. Instantaneous power requirements of the body and its various divisions at different walking speeds.

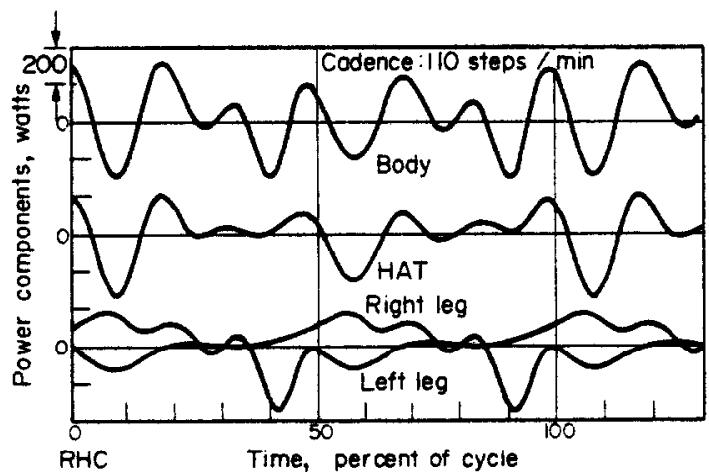

Fig. 5. Contribution of major body components to the total instantaneous power of the body.

pattern as described in Zarrugh and Radcliffe (1979). The speed was kept constant at $1.50 \mathrm{~m} / \mathrm{sec}$ while the step rate was varied from 90 to 140 steps/min. While the mean energy level of the body decreases slowly with increasing step rate, the changes about the mean value are essentially identical for all cadences. The average energy level of the HAT decreases also with cadence but the changes in ME decrease with step rate (Fig. 6b).

The lower limb exhibits little change in its energy level pattern as cadence increases (Fig. 6c). Although at heel contact the right thigh has a decreasing energy level as step rate increases, shapes of the energy curves are similar for all step rates (Fig. 6d). Figures 6(e) and (f) indicate that the energy levels of the shank and the foot are influenced very little by changes in cadence.

\section{Power requirements of segments in forced walking}

The power required to accelerate and decelerate the body and its components are shown in Figs. 7(a)-(f). Minor effects of varying the cadence on the power of the body are observed. Some cadence-related changes do occur in the case of the HAT (Fig. 7b) and the right lower limb (Fig. 7c), but the amount of energy removed from or added to the body per cycle is essentially the same regardless of cadence (Fig. 7a). In all lower limb segments (Figs. $7 d$, e and $f$ ), the mean positive requirements increase with increased step rate.

\section{Mean power needs and efficiency in free walking}

In level walking at a steady speed, the energy level of any body segment is constantly changing; internal muscular work is the sole source of these changes. No external work can be done in level walking because the ground reactions do no work and the force of gravity is represented by the potential energy component. Because level walking is cyclical, the net ME change in any segment during one complete cycle is essentially zero. Hence, positive work done per cycle will always 

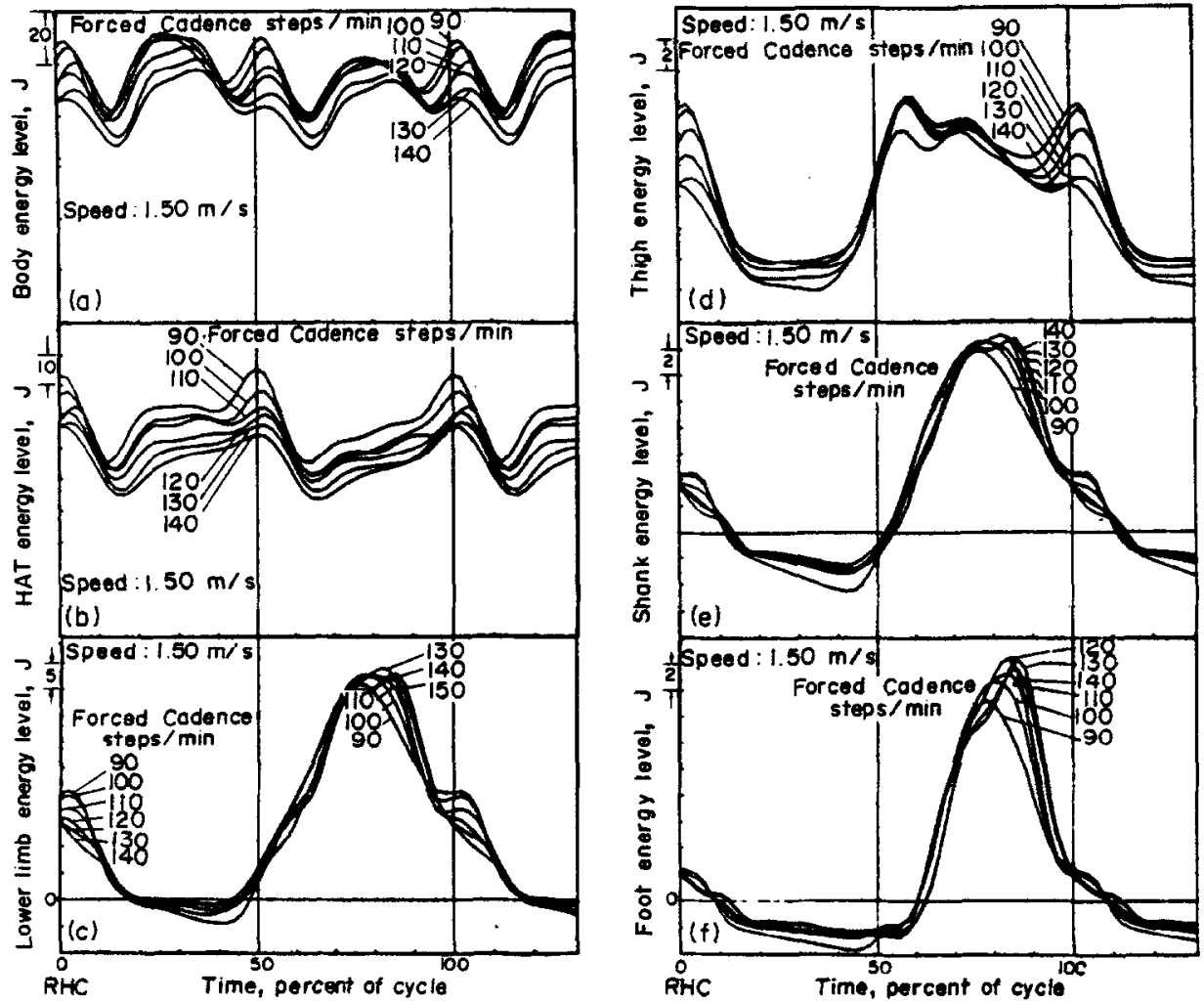

Fig. 6. Energy levels as a function of forced cadence at a constant speed of $1.5 \mathrm{~m} / \mathrm{sec}$.
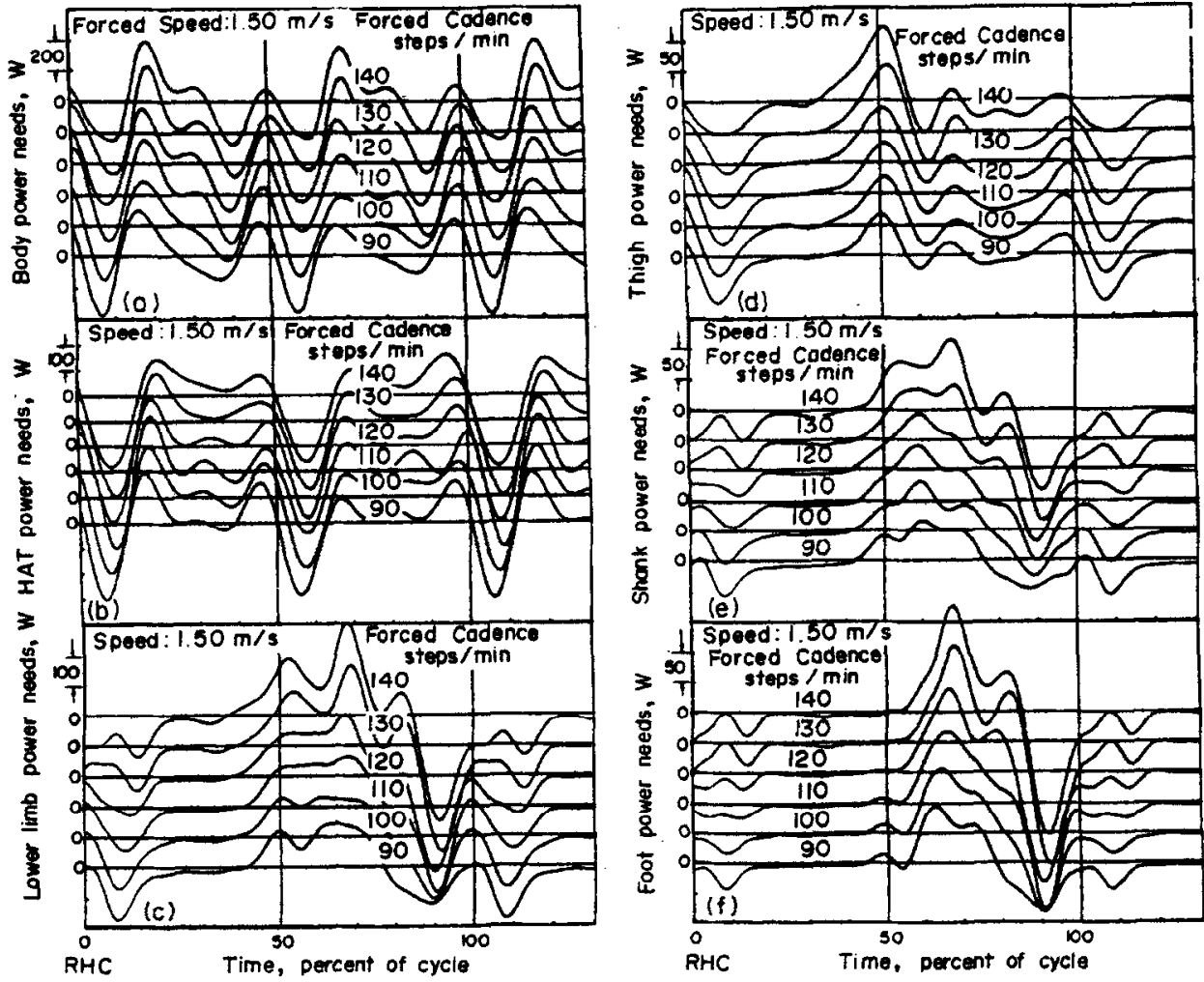

Fig. 7. Power requirements of walking with different forced cadences at a constant speed of $1.5 \mathrm{~m} / \mathrm{sec}$. 
equal the negative work done in cyclical movement. Although the net work done in this case is zero, muscular effort is still required to bring about the irreversible changes in the energy level. The larger portion of the metabolic power appearing as work fuels the shortening of the muscles which generally accelerates the segments relative to each other. Negative work dissipated by the forced lengthening of active muscles also requires muscular effort but the metabolic cost of negative work is at best one-fifth that of positive work (Abbott et al., 1952; Davies and Barnes, 1972). Therefore, the rate of positive work or positive power is the most physiologically significant quantity. It is a simple matter, however, to consider negative work, as well as positive work, since the two are equal in mean magnitude.

The average positive work rates required to increase $\mathrm{ME}, \mathrm{KE}$ and PE of the body segments, as well as the body as a whole, are displayed in Fig. 8. As a consequence of exchanges between kinetic and potential energy within segments, the total work rate, $\dot{W}_{t}$, is considerably smaller than the sum of potential energy work, $\dot{W}_{p}$, and kinetic energy work, $\dot{W}_{k}$. This indicates that some of the changes in PE are recovered as changes in $\mathrm{KE}$ and vice versa, but the recovery is not complete since the total work rate is not zero. The average total work rate of the body, $W_{t}$, is shown in Fig. 8 to vary approximately in a linear form with speed. The rate of change of body's PE, $W_{p}$, and KE, $\dot{W}_{k}$, both increase monotonically with speed.

Figure 9 displays the effects of walking speed on the total work rate of the HAT, lower limb and lower limb segments. The work rates for these divisions are shown to vary approximately as the speed squared. The sum of work rates of the component parts does not equal the work rate of the whole because of energy exchange between the component parts. For example, at $1.5 \mathrm{~m} /$ $\mathrm{sec}$, the sum of the work rates for the shank, thigh and foot is $47.3 \mathrm{~W}$, while the average rate for the limb as a whole is $39.0 \mathrm{~W}$. An average power of $8.3 \mathrm{~W}$ is eliminated from the amount which would be required

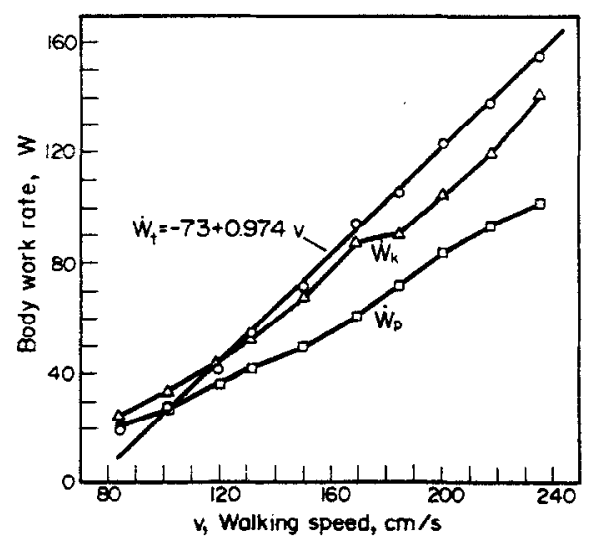

Fig. 8. Average work rates to increase the total, kinetic and potential energy of the body as a function of speed in free walking.

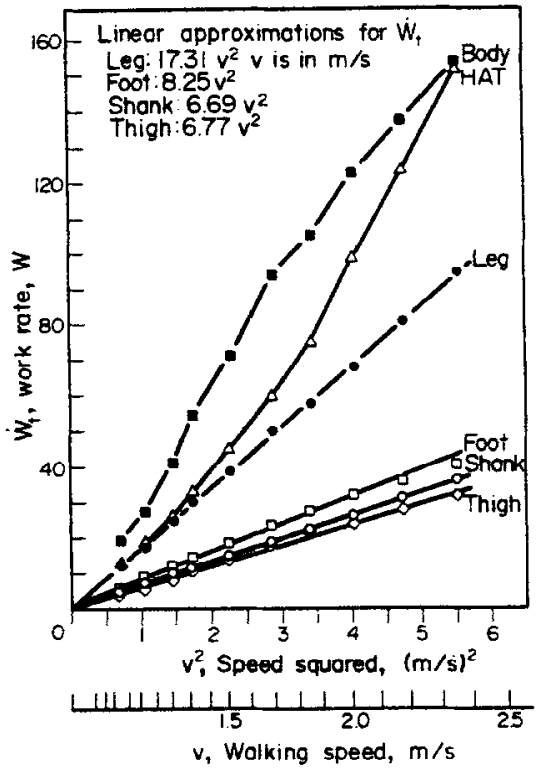

Fig. 9. Total work rates of various body segments as a function of speed squared in free walking.

if no energy exchange takes place. At the same speed, the true power requirements of the body which take exchanges between segments in consideration are $71.4 \mathrm{~W}$, while the sum of the average total work rates of the individual segments (i.e. no exchange between segments) is $140.2 \mathrm{~W}$.

Since both positive and negative work require the expenditure of metabolic energy, they both must be included in any measure of efficiency. However, no consistent reports of the relative cost of the two work types exist in the literature. If the cost of performing negative work is assumed to be zero, as is done in this paper, minimum efficiency values are predicted. On the other hand, if equal costs are assigned, as suggested by * Winter (1979), upper extreme efficiency values are obtained. Efficiency computed in this manner will be twice as large as the minimum values predicted when negative work is excluded. The true efficiency lies within these two extremes. For example, if the relative cost of positive to negative work is 5 to 1 , the efficiencies reported in this paper are underestimated by $20 \%$. These efficiencies are also low because they do not reflect isometric muscular effort to hold limbs against gravity or muscular co-contractions when positive work produced by one muscle is absorbed by an antagonistic muscle.

Considering positive work only, Fig. 10 shows that the efficiency increases rapidly from $9 \%$ at $0.84 \mathrm{~m} / \mathrm{sec}$ to the maximum value of $22.7 \%$ at $1.70 \mathrm{~m} / \mathrm{sec}$ and it slowly decreases at higher speeds to $18.5 \%$ at $2.35 \mathrm{~m} /$ $\mathrm{sec}$. Bobbert (1960) listed values ranging from 11.1 to $27 \%$ for walking, cranking and cycling. Fenn (1930a) reported an efficiency of $23 \%$ for his subjects in running. In walking, the subject of Ralston and Lukin (1969) showed an average efficiency of $22 \%$. The 


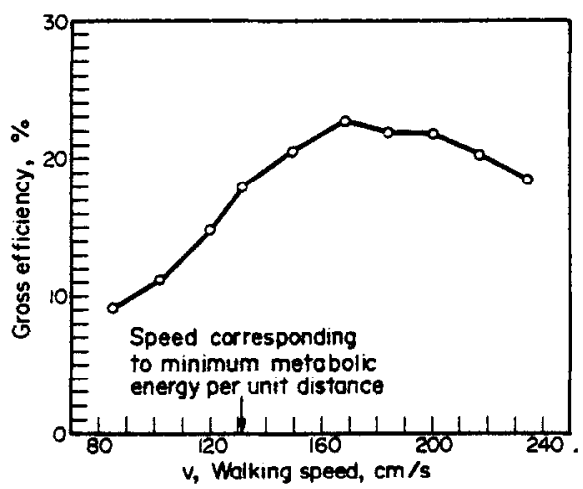

Fig. 10. Variation of gross efficiency with speed in free walking.

increase in gross efficiency with work load is well documented in the literature (Bobbert, 1960). However, its decrease at very high loads has not been reported previously. Considering the absolute energy changes of segments (equal metabolic cost is assigned to positive and negative work), Norman et al. (1976) have shown for treadmill running that many efficiency values ranging from 12 to $34 \%$ can be obtained, if different definitions were used for the mechanical work output and metabolic input. They recommended that the metabolic input be represented by the net (i.e. gross less pre-exercise) aerobic plus anaerobic oxygen consumption and the mechanical output by the absolute value of the total work rate of the body.

\section{Mean power and efficiency in forced walking}

The average work rates of various body segments during forced walking are reported in Fig. 11. Figure 11 shows that if the step rate is varied while the speed remains constant, the average positive work rate of the body (open circles, top curve) remains essentially constant at a level equal to the work rate required by the body in walking at the same speed but with the free step rate (dashed line in Fig. 11). This condition of constant work rate results in a maximum gross efficiency (lower continuous curve and open squares, Fig. 11) at the freely chosen step rate since it requires the least metabolic energy rate (top continuous curve, Fig. 11). Thus, when the speed is prescribed, a combination of step length and step rate that requires the least metabolic power and hence, produces maximum mechanical efficiency is automatically selected. The work rate needed to increase the energy level of the lower limb increases linearly with step rate (Fig. 12). The same figure shows the work rate of the HAT to have a minimum near the free step rate of about $\mathbf{1 1 0}$ steps/min which corresponds to this speed of $1.50 \mathrm{~m} /$ sec. This supports the assertion made by Bresler et al. (1957) that the reduction of the irregularity of trunk motion might result in substantial reductions in the energy needs of amputees.

Acknowledgements - This work was supported in part by Veterans Administration Contract VAV 101(134).

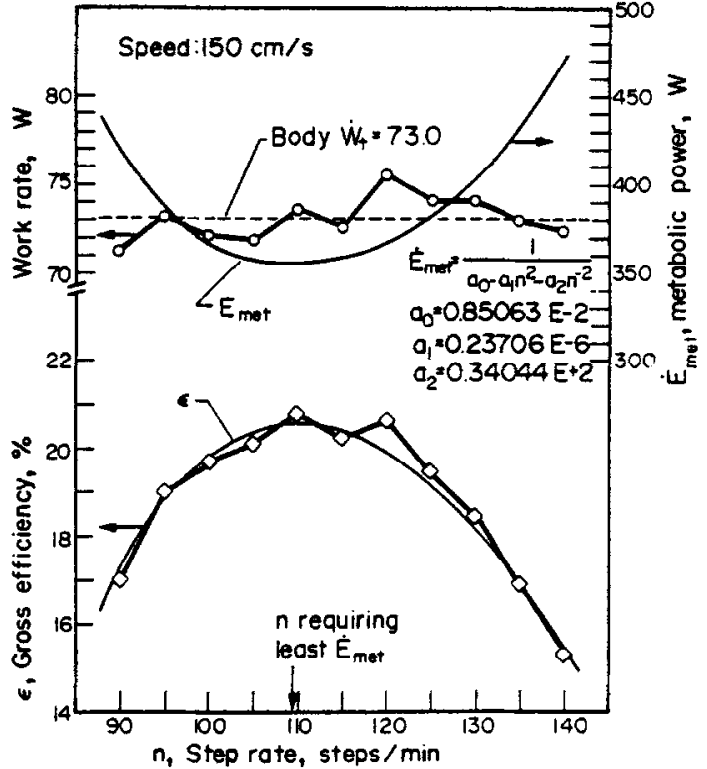

Fig. 11. Work rate, metabolic power and efficiency as a function of forced step rate at the same constant speed.

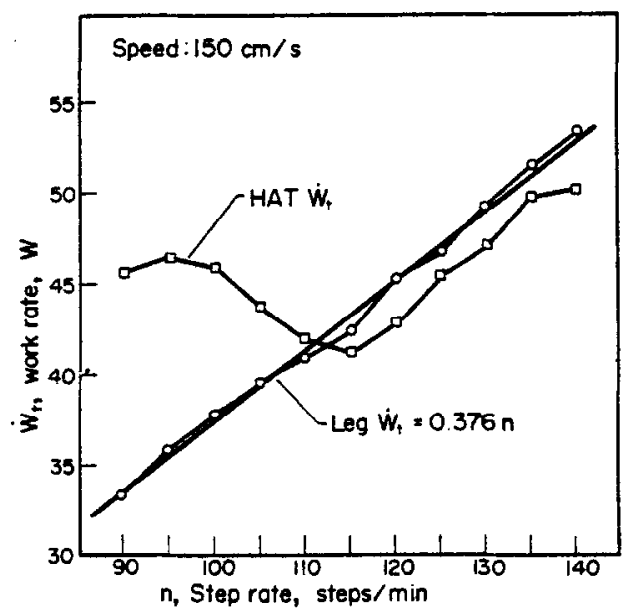

Fig. 12. Variation of the work rate of the HAT and the right lower limb with forced step rate-

\section{REFERENCES}

Abbott, B. C., Bigland, B. and Ritchie, J. M. (1952) The physiological cost of negative work. J. Physiol. Lond. 117, 380-390.

Bobbert, A. C. (1960) Physiological comparison of three types of ergometry. J. appl. Physiol. 15, 1007-1014.

Bresler, B. and Berry, F. R. (1951) Energy and power in the leg during level walking. Prosthetic Devices Research Project, Institute of Engineering Research, University of California, Berkeley, Series II, Issue 15.

Bresier, B., Radcliffe, C. W. and Berry, F. R. (1957) Energy and power in the legs of above-knee amputees during normal level walking. Prosthetic Devices Research Project, Institute of Engineering Research, University of California, Berkeley, Series II, Issue 31.

Cavagna, G. A. (1975) Force platforms as ergometers. J. appl. Physiol. 39, 174-179. 
Cavagna, G. A., Saibene, F. R. and Margaria, R. (1963) External work in walking. J. appl. Physiol. 18, 1-9.

Cavagna, G. A. and Margaria, R. (1966) Mechanics of walking. J. appl. Physiol. 21, 271-278.

Davies, C. T. M. and Barnes, C. (1972) Negative (eccentric) work. II : Physiological responses to walking uphill and downhill on a motor-driven treadmill. Ergonomics 15, 121-131.

Elftman, H. (1939) Forces and energy changes in the leg during walking. Am. J. Physiol. 125, 339-356.

Elftman, H. (1940) The work done by muscles in running. Am. J. Physiol. 129, 672-684.

Elftman, H. (1955) Knee action and locomotion. Bull. Hosp. Diseases 16, 103-110.

Fenn, W.O. (1930a) Frictional and kinetic factors in the work of sprint running. Am. J. Physiol. 92, 538-611.

Fenn, W. O. (1930b) Work against gravity and work due to velocity changes in running. Am. J. Physiol. 93, 433-462.

Gersten, J. W, Orr, W., Sexton, A. W. and Okin, D. (1969) External work in level walking. J. appl. Physiol. 26, 286-289.

Lamoreux, L. W. (1971) Kinematic measurement in the study of human walking. Bull. Prosthetics Res. 10(10-15), 3-84.

Lukin, L., Polissar, M. J. and Ralston, H. J. (1967) Methods of studying energy costs and energy flow during human locomotion. Human Factors 9, 603-608.

Norman, R. W., Sharratt, M. T., Pezzack, J. C. and Noble, E. G. (1976) Re-examination of the mechanical efficiency of horizontal treadmill running. Biomechanics VB (Edited by Komi, P. V.), Vol. 1B, pp. 87-93. University Park Press, Baltimore.

Ralston, H. J. and Lukin, L. (1969) Energy levels of human body segments during level walking. Ergonomics 12, 39-46.

Winter, D. A. (1979) A new definition of mechanical work done in human movement. J. appl. Physiol. 46, 79-83.

Winter, D. A., Quanbury, A. O. and Reimer, G. D. (1976) Analysis of instantaneous energy of normal gait. J. Biomech. 9, 253-257.

Zarrugh, M. Y. (1976) Energy considerations in human walking. Fedn Proc. Fedn Am. Socs exp. Biol. 35(3), 529.

Zarrugh, M. Y. (1978) Mechanical efficiency in walking. Proc. Second Annual Meeting Am. Soc. Biomech., October 1978, Ann Arbor, MI. Also published in J. Biomech. 12, 633 (1979).

Zarrugh, M. Y. and Radcliffe, C. W. (1978) Predicting metabolic cost of level walking. Eur. J. appl. Physiol. 38, 215-223.

Zarrugh, M. Y. and Radclifie, C. W. (1979) Computer generation of human gait kinematics. J. Biomech. 12, 99-111. 